\title{
Retrospective cohort study of patients qualified for lung transplantation due to idiopathic pulmonary fibrosis - single-centre experience
}

\author{
Marek Ochman ${ }^{1}$, Maciej Urlik ${ }^{1}$, Zofia Tatoj ${ }^{1}$, Fryderyk Zawadzki ${ }^{1}$, Marta Wajda-Pokrontka ${ }^{1}$, \\ Magdalena Latos ${ }^{1}$, Piotr Przybyłowski²,3, Marian Zembala ${ }^{1}$
}

\begin{abstract}
${ }^{1}$ Department of Cardiac, Vascular and Endovascular Surgery and Transplantology in Zabrze, Medical University of Silesia in Katowice, Poland

${ }^{2} 1^{\text {st }}$ Chair of General Surgery, Jagiellonian University Medical College, Krakow, Poland ${ }^{3}$ Silesian Center for Heart Diseases, Zabrze, Poland
\end{abstract}

Submitted: 22 November 2018

Accepted: 14 January 2019

Arch Med Sci 2020; 16 (3): 621-626

DOI: https://doi.org/10.5114/aoms.2019.82662

Copyright (c) 2019 Termedia \& Banach

\section{Abstract}

Introduction: Idiopathic pulmonary fibrosis (IPF) is a chronic and progressive disease. Pharmacological treatment can only slow its progression. However, lung transplantation (LTX) is the only treatment for patients with its endstage form. This study analysed the long-term results of the qualification process of patients with IPF recruited for LTx in a single centre.

Material and methods: Retrospective analysis of 84 patients (56 patients who died while on the waiting list and 28 patients who underwent LTX) with end-stage IPF who were qualified for LTx between 2006 and 2017 at the Silesian Centre for Heart Diseases (Zabrze, Poland).

Results: Cox proportional hazard analysis showed that the only parameter was 6-minute walk test (6MWT) distance, which statistically significantly impacted the probability of receiving a graft (parameter assessment, 0.00523; $p=0.006$; $95 \%$ confidence interval $(\mathrm{Cl}): 0.0015-0.009$; hazard ratio $(\mathrm{HR})=$ $1.005)$ as well as that of death while on the waiting list (parameter assessment, $-0.0054 ; p=0.003 ; 95 \% \mathrm{Cl}:-0.009-(-0.0017) ; \mathrm{HR}=0.995)$. Patients with a 253-350-m 6MWT distance had 3 times greater risk of dying while on the waiting list than those who walked more than $350 \mathrm{~m}$. Other factors, such as height, sex, and blood group, also influenced the outcome.

Conclusions: The 6-minute walk test distance is an independent predictor of mortality on the lung transplant waiting list. Blood type and height also play a significant role in becoming a lung recipient.

Key words: idiopathic pulmonary fibrosis, interstitial lung diseases, lung transplantation, qualification, mortality.

\section{Introduction}

Idiopathic pulmonary fibrosis (IPF) is the most common form of chronic, progressive interstitial lung disease (ILD) [1]. Characterized by growth of fibrotic tissue in the parenchyma of the lungs, IPF is associated with high morbidity and poor prognosis [2]. Patients become less and less self-reliant, finally being dependable on other people or home health services [3]. The mean survival time for patients with IPF ranges from 3 to 5 years. In Europe, the incidence of IPF was 3-9 cases per 100,000 people in 2017 and is increasing [4]. The radiographic findings of subpleural re-

\author{
Corresponding author: \\ Marek Ochman MD, PhD \\ Department of Cardiac, \\ Vascular and \\ Endovascular Surgery \\ and Transplantology \\ Medical University \\ of Silesia \\ 9 M. Curie-Skłodowskiej St \\ 41-800 Zabrze, Poland \\ Phone/fax: +48 324793483 \\ E-mail:mochman@sccs.pl
}


ticulation, honeycombing, and bronchiectasis are typical for IPF [5]. Its progression is characterized by lowered diffusion capacity for carbon monoxide (DLCO), forced vital capacity (FVC), and total lung capacity (TLC) in pulmonary function tests [5]. Although the pathogenesis of IPF has been studied for more than 20 years, it still remains unclear $[6,7]$. Risk factors that have been associated with the outbreak of IPF are cigarette smoking and exposure to wood, metal, stone, and organic dust [7]. The IPF is diagnosed only after excluding other ILDs [8]. Clinical presentation of IPF is not distinctive among ILDs [9]. Pirfenidone (antifibrotic drugs) and nintedanib (multityrosine kinase inhibitor) can slow the progression of IPF, so they are used in the treatment of IPF [1]. However, the only treatment that is proven to help the patients is lung transplantation (LTX) [10]. During the qualification process, in accordance with the International Society of Heart and Lung Transplantation (ISHLT) guidelines, the following clinical findings are taken into consideration:

- Decline in FVC $\geq 10 \%$ during 6 months of follow-up (note: a $5 \%$ decline is associated with a poorer prognosis and may warrant listing);

- Decline in DLCO $\geq 15 \%$ during 6 months of follow-up;

- Oxygen desaturation to $<88 \%$, a 6-minute walk test (6MWT) distance $<250 \mathrm{~m}$, or a $>50 \mathrm{~m}$ decline in 6MWT distance over a 6-month period;

- Pulmonary hypertension on right heart catheterization or 2-dimensional echocardiography;

- Hospitalization because of respiratory failure, pneumothorax or acute exacerbation [11].

The first successful LTx with long-term survival was performed in 1983 at the University of Toronto. The recipient was a patient with IPF. Since then, LTx has become an established method of treatment for patients with end-stage IPF [10]. Systems of lung allocation vary across different lung transplant programmes. It is worth remembering that each allocation system favours different potential recipients.

The aim of this study was to analyse the longterm results of the qualification process of patients with IPF recruited for LTx in a single centre.

\section{Material and methods}

\section{Study population}

This retrospective cohort study analysed clinical records of 84 patients with IPF. Its population comprised patients with the end stage of this lung disease who were qualified to undergo LTx according to the ISHLT guidelines for potential recipients (30 women, 54 men; median age at qualification, 51.11 years; range: $18-65$ years). The study group included 56 (63.53\%) patients who died while on the waiting list (25 women, 31 men; median age at qualification: 52.37 years; range: $18-65$ years) and 28 patients who underwent LTx (32.94\%) (5 women, 23 men; median age: 48.83 years; range: 25-60 years) between 2006 and 2017 in the Silesian Centre for Heart Disease (Zabrze, Poland). Qualified patients with unknown end-points (neither undergoing LTx nor dying while on the national LTx waiting list) were excluded from the study.

\section{Data collection}

Data regarding the qualification for LTX were collected from the medical records and potential recipient questionnaires. The following basic characteristics were analysed: age (years), sex, height $(\mathrm{cm})$, weight $(\mathrm{kg})$, body mass index $\left(\mathrm{kg} / \mathrm{cm}^{2}\right)$, and time spent on the waiting list (days). During the qualification process, kidney function (serum creatinine $(\mathrm{mg} / \mathrm{dl})$, estimated glomerular filtration rate $\left(\mathrm{ml} / \mathrm{min}^{-1} / 1.73 \mathrm{~m}^{2}\right)$ ), liver function (serum bilirubin $(\mathrm{mg} / \mathrm{dl})$, aspartate transaminase (UI/l), alanine transaminase $(\mathrm{UI} / \mathrm{l})$ ), lipid profile, full blood count and $\mathrm{N}$-terminal prohormone of brain natriuretic peptide (NT-proBNP) levels $(\mathrm{pg} / \mathrm{ml})$ were evaluated in laboratory tests. Pulmonary function tests, such as spirometry (forced expiratory volume in $1^{\text {st }} \mathrm{S}\left(\mathrm{FEV}_{1} \%\right)$, actual $\mathrm{FEV}_{1}(\mathrm{l})$, and forced vital capacity (FVC\%)), as well as arterial blood gas assessment, were performed. Further, 6MWT distance $(\mathrm{m})$, Borg's scale, and oxygen saturation $\left(\mathrm{SpO}_{2}(\%)\right)$ were analysed. Cardiac evaluation included echocardiography (left ventricle ejection fraction (\%), right ventricular systolic pressure $(\mathrm{mm} \mathrm{Hg})$, tricuspid annular plane systolic excursion ( $\mathrm{mm}$ ), and acceleration time (ms)) and right heart catheterization (mean artery pulmonary pressure $(\mathrm{mm} \mathrm{Hg})$ ). The following lung plethysmography parameters were also assessed: predicted total lung capacity (TLC) (I) and residual volume (RV) (\%).

\section{Statistical analysis}

Data were expressed as mean \pm standard deviation. The Mann-Whitney $U$-test, $\chi^{2}$ test with Yates' correction, and maximum likelihood chisquared test were used, as appropriate. Statistica 10.0 software (StatSoft, Inc., Tulsa, OK, USA) was used for statistical analysis. All findings with $p<0.05$ were considered statistically significant. The results in certain tables were presented as hazard ratios (HRs) with 95\% confidence intervals (Cls). Cox proportional hazard analysis was used for evaluation of the effect of continuous and dichotomous data on the incidence of death. Considering their collinearity, statistically significant variables from the single factor analysis $(p<0.05)$ were included in the multiple factor analysis using Cox proportional hazard analysis. 


\section{Results}

Statistically significant findings regarding patients' results during qualification for LTx are presented in Table I. Significant differences between deceased patients and those who underwent LTX were observed in the following parameters: per-

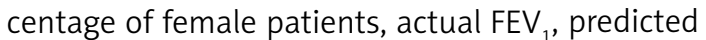
TLC, 6MWT distance and height. It was statistically significant that patients shorter than $160 \mathrm{~cm}$ had poorer chances of finding a suitable donor. This finding is particularly important among female candidates, as a statistically significant difference was noted between their percentages in the two studied groups. Taller people are also characterised by proportionally greater total lung capacity. Therefore, height also influenced the aforementioned parameter, as a significant difference between studied groups was distinguished.

Patients who underwent LTx were on the waiting list for 35 days (on average) less than the deceased ones. Both groups had similar age, weight, and body mass index. Laboratory tests revealed good functional state of the kidneys and liver as well as elevated NT-proBNP level in both the groups. Lipid profile and full blood count parameters did not differ statistically significantly between studied groups.

Pulmonary functional tests, including spirometry, showed reduced parameter values (FEV $\%$ and FVC \%) among patients who died while on the waiting list. The group of patients who underwent LTX (FVC\%: 50.86, FEV \%: 51.16) showed better results than their deceased counterparts (FVC\%: 42, $\mathrm{FEV}_{1} \%$ : 44.66). A significant difference was observed in actual FEV between the two groups. Arterial blood gas tests revealed respiratory failure in $66.22 \%$ of the patients $(40.74 \%$ of the deceased patients, $85 \%$ of those who underwent LTx); type 1 respiratory failure was observed in $43.24 \%$ of the patients $(37.04 \%$ of the deceased patients, $60 \%$ of those who underwent LTX) and type 2 was observed in $22.97 \%$ of the patients $(22.22 \%$ of the deceased patients, $25 \%$ of those who underwent LTX).
Patients who underwent LTx achieved $169 \mathrm{~m}$ more on average during the 6MWT than their deceased counterparts; this difference was statistically significant (Table I). Both groups perceived exertion approximately at the level of 4 according to Borg's scale.

Echocardiography revealed normal left ventricle ejection fraction, concurrent with features of increased resistance in pulmonary circulation. During right heart catheterization, pulmonary hypertension was observed in $71.43 \%$ of the patients with IPF $(72.73 \%$ of the deceased patients and $66.67 \%$ of those who underwent LTX).

Univariate analysis was performed to determine which of the parameters were statistically significant factors for becoming a lung recipient. Results of the aforementioned analysis are presented in Table II. Significant factors associated with undergoing LTx were as follows: actual FEV , $_{1}$ actual TLC, 6MWT distance, height, residual volume as a percentage of the predicted value, and blood type. Patients with blood type $A B$ are approximately 3 times more likely to become graft recipients in comparison to other blood types ( $p=0.003$; 95\% Cl: 0.009-2.32; HR = 3.32). Taller patients have greater predicted values of $\mathrm{FEV}_{1}$ and TLC. Therefore, they will have greater results of actual amounts in litres of described parameters in comparison to their shorter counterparts presenting the same percentage of these values. Risk factors associated with higher probability of death while on the LTx waiting list were also determined using univariate analysis. Statistically significant results are presented in Table III. Patients with blood type $B$ and those who achieved a shorter distance in the 6MWT were more likely to present an unfavourable outcome while on the waiting list. Stepwise regression analysis was used to reduce the multivariate model to a single parameter: the 6MWT distance. This parameter significantly impacts the probability of becoming a lung recipient (parameter assessment, $0.00523 ; p=0.006$; $95 \% \mathrm{Cl}$ : 0.0015-0.009;

Table I. Statistically significant results during qualification for lung transplantation

\begin{tabular}{|c|c|c|c|c|}
\hline Parameter & $\begin{array}{l}\text { All qualified patients } \\
\qquad(n=84)\end{array}$ & $\begin{array}{l}\text { Patients deceased } \\
\text { on waiting list } \\
(n=56)\end{array}$ & $\begin{array}{c}\text { Transplanted } \\
\text { patients }(n=28)\end{array}$ & $P$-value \\
\hline Sex, female, \% & 35 & 44.64 & 12.5 & $0.01^{b}$ \\
\hline Height, mean (SD) [cm] & $167.54(9.548)$ & $165.49(9.874)$ & $172.31(6.785)$ & $0.004^{\mathrm{a}}$ \\
\hline Height $<160 \mathrm{~cm}(\%)$ & 23.75 & 32.14 & 4.17 & $0.02^{b}$ \\
\hline $\mathrm{FEV}_{1}$ actual, mean (SD) [l] & $1.47(0.659)$ & $1.35(0.621)$ & $1.78(0.672)$ & $0.02^{a}$ \\
\hline TLC predicted, mean (SD) [I] & $6.07(1.395)$ & $5.8(1.410)$ & $7.14(0.652)$ & $0.03^{a}$ \\
\hline 6MWT distance, mean (SD) [m] & $227.22(156.452)$ & $176.57(136.845)$ & $346.24(135.764)$ & $0.0005^{a}$ \\
\hline
\end{tabular}


Table II. Univariable analysis of factors associated with being transplanted

\begin{tabular}{|lcccccc|}
\hline Parameter & $\begin{array}{c}\text { Parameter } \\
\text { assessment }\end{array}$ & SE & $\chi^{2}$ test & P-value & $95 \%$ Cl & HR \\
\hline Height [cm] & 0.10 & 0.03 & 12.46 & 0.0004 & $0.04-0.15$ & 1.10 \\
\hline FEV ${ }_{1}$ actual [I] & 0.92 & 0.37 & 6.35 & 0.01 & $0.20-1.64$ & 2.51 \\
\hline Distance [m] & 0.01 & 0.002 & 8.52 & 0.004 & $0.002-0.01$ & 1.01 \\
\hline TLC actual [I] & 0.60 & 0.22 & 7.28 & 0.007 & $0.16-1.03$ & 1.81 \\
\hline RV, \% of predicted value & 0.03 & 0.01 & 6.44 & 0.01 & $0.01-0.05$ & 1.03 \\
\hline Blood type O & -0.72 & 0.49 & 2.16 & 0.1 & $-1.67-0.24$ & 0.49 \\
\hline Blood type A & 0.16 & 0.44 & 0.13 & 0.7 & $-0.71-1.03$ & 1.17 \\
\hline Blood type B & 0.02 & 0.76 & 0.0004 & 1.0 & $-1.47-1.50$ & 1.02 \\
\hline Blood type AB & 1.20 & 0.57 & 4.46 & 0.03 & $0.09-2.32$ & 3.32 \\
\hline
\end{tabular}

$\mathrm{Cl}$ - confidence interval, $\mathrm{FEV}$ - forced expiratory volume in $1^{\text {st }} \mathrm{S}$, HR - hazard ratio, $R V$ - residual volume, TLC - total lung capacity. A $p$-value of $<0.05$ is considered statistically significant.

Table III. Univariable analysis of factors associated with death while on the waiting list for lung transplantation

\begin{tabular}{|lcccccc|}
\hline Parameter & $\begin{array}{c}\text { Parameter } \\
\text { assessment }\end{array}$ & SE & $\chi^{2}$ test & P-value & $95 \% \mathrm{Cl}$ & HR \\
\hline Distance [m] & -0.01 & 0.001 & 15.49 & 0.00008 & $-0.01--0.003$ & 0.99 \\
\hline Blood type O & -0.34 & 0.29 & 1.37 & 0.2 & $-0.91-0.23$ & 0.71 \\
\hline Blood type A & -0.07 & 0.29 & 0.05 & 0.8 & $-0.64-0.51$ & 0.94 \\
\hline Blood type B & 0.95 & 0.39 & 5.91 & 0.02 & $0.18-1.71$ & 2.58 \\
\hline Blood type AB & 0.03 & 0.60 & 0.003 & 1.0 & $-1.15-1.21$ & 1.03 \\
\hline
\end{tabular}

Cl-confidence interval, $H R$ - hazard ratio. A p-value of $<0.05$ is considered statistically significant.

$H R=1.005)$ as well as that of death while waiting for LTx (parameter assessment, $-0.0054 ; p=0.003$; $95 \% \mathrm{Cl}:-0.009-(-0.0017) ; \mathrm{HR}=0.995)$. Due to these findings, quantile division of the aforementioned parameter was performed $\left(1^{\text {st }}\right.$ quantile: $\leq 72 \mathrm{~m}, 2^{\text {nd }}: 72-252 \mathrm{~m}, 3^{\text {rd }}: 253-350 \mathrm{~m}$, and $4^{\text {th }}$ : $>350 \mathrm{~m}$ ). Further analysis revealed that all qualified patients whose 6MWT distance was within the $1^{\text {st }}$ quantile died before LTx. However, the patients whose 6MWT distances were between the $2^{\text {nd }}$ and $3^{\text {rd }}$ quantiles had smaller probabilities of undergoing LTX (respectively $28.1 \%$ and $27.7 \%$ ) than those with 6MWT distances in the $4^{\text {th }}$ quantile. The same quantile division was used to assess the risk of death while on the LTx waiting list. The analysis revealed that patients from the $1^{\text {st }}$ quantile had 10 times greater probability of dying without LTx in comparison to those from the $4^{\text {th }}$ quantile. Patients from the $3^{\text {rd }}$ quantile were 3 times more likely to die than those from the $4^{\text {th }}$ one.

Twenty-eight patients $32.94 \%$ of all qualified patients) underwent LTx. This group included 23 (82.14\%) men and $5(17.86 \%)$ women. Twenty (71.43\%) patients underwent SLT and the remaining $8(28.57 \%)$ patients underwent DLT. Mean age at the day of LTX was 50.58 years.

\section{Discussion}

The IPF is a disease of unknown origin, leading to complete respiratory insufficiency. It was demonstrated by Brown et al. that patients with higher BMI, FVC \% predicted, FEV $\%$ predicted, TLC \% predicted, and diffusing capacity of lung for carbon monoxide \% predicted, but a lower $\mathrm{FEV}_{1} / \mathrm{FVC}$ ratio and lower mean pulmonary artery pressures, had a better chance of reaching 5-year survival [12]. This finding is confirmed by Castria et al., whose work proved that patients with IPF had a better chance of survival when their pulmonary artery pressure was low [13]. As a viable treatment option, LTx improves lung function in patients with IPF. Multivariate analysis showed that $6 \mathrm{MWT}$ distance is the most significant factor influencing the patients' outcome. However, other factors, such as height, blood type and particular results of pulmonary function tests, may influence the outcome of patients. Our study showed that blood type $A B$ was associated with greater probability of becoming a graft recipient whereas blood type $O$ was related to dying while on the waiting list. This aspect was also studied by Mackay et al., who found that patients with blood type A or B 
are more likely to undergo transplantation than patients with blood type $\mathrm{O}[14]$.

In the lung transplantation programme at the Silesian Center for Heart Diseases, 102 patients with end-stage IPF qualified to undergo LTx between 2006 and 2017. Eighty-four of these patients were included in this study, as they had reached an end-point (LTX or death while on the waiting list). It is worth mentioning that down-sizing of the donor lungs is an important part of donor-recipient matching due to the fibrotic changes in the lungs that reduce the thoracic volume of the recipient. The average height of men and women in Poland is $178.7 \mathrm{~cm}$ and $165.1 \mathrm{~cm}$, respectively, which implies that taller patients have a greater chance of finding a suitable donor sooner [15]. The two studied groups differed significantly in terms of height: deceased patients were 165.49 $\mathrm{cm}$ tall on average. Lung recipients were taller, with a mean height of $172.31 \mathrm{~cm}$.

A significant difference was observed in the distance achieved during the 6MWT between the two studied groups. Patients who underwent LTX achieved $346.24 \mathrm{~m}$ on average. Deceased patients were able to walk less on average (191.21 m). A comparable level of fatigue (according to the subjective Borg's scale) was noted in both groups. It is worth mentioning that 6MWT distance has been established to be predictive of mortality among patients qualified for LTx [10]. This finding was also confirmed by our study, as 6MWT was found to have a significant predictive impact on the probability of undergoing LTX, as indicated by Cox proportional hazard analysis. Patients who achieved a 6MWT distance between 253 and $350 \mathrm{~m}$ had 3 times greater probability of dying while on the waiting list in comparison to patients who achieved more than $350 \mathrm{~m}$. Lederer et al. published a study indicating that a low result obtained in the 6MWT was strongly and independently associated with an increased mortality rate. Patients with a walking distance less than $207 \mathrm{~m}$ had a more than fourfold higher mortality rate than those with a walking distance of $207 \mathrm{~m}$ [16]. According to the ISHLT guidelines, in order to qualify, patients have to obtain a 6MWT distance less than $250 \mathrm{~m}$ or show a decrease of at least $50 \mathrm{~m}$ in this parameter across 6 months [11]. It was proved by du Bois et al. that baseline 6MWT distance $<250 \mathrm{~m}$ was associated with a two-fold increase in the risk of mortality and a 24-week decline in 6MWT distance $>50 \mathrm{~m}$ was associated with a nearly three-fold increase in mortality risk [17]. It is the authors' opinion that it is worth considering sending patients to begin the qualification process sooner, when their 6MWT distance is less than $350 \mathrm{~m}$. This might ultimately increase their chances of receiving the graft. Taller patients are known to achieve longer 6MWT distances.
Therefore, we took this into consideration but did not find that taller patients achieved longer 6MWT distances. Ikezoe et al. published a study assessing prognostic factors and outcomes of lung transplant candidates due to ILD [18]. Their multiple stepwise analysis showed that 6MWT distance also independently contributed to mortality of all ILD patients. An additional investigation focused solely on IPF patients $(n=33)$ supported by a stepwise multivariate Cox proportional hazards model revealed that $\mathrm{BMI}$ and a history of acute exacerbation independently contributed to increased mortality while waiting for a lung transplantation. On the other hand, a study published by Bennett et al. presented results contradicting those of Ikezoe and ours [19]. Their research demonstrated that reduced survival was associated with higher levels of partial pressure of carbon dioxide in arterial blood despite average values being within the normal range. Finally, Mackay et al. analysed IPF mortality among patients awaiting LTX, concluding that disease progression is a more sensitive indication for LTX referral than any physiological measure of disease severity [14]. Lipid profile did not differ between the two studied groups, which is consistent with the finding that plasma lipid levels had a neutral influence on the occurrence of the outcomes [20]. Twenty percent of the patients receiving lung graft between 2006 and 2017 underwent LTX due to IPF in the Silesian Center for Heart Diseases. This makes it the second most common group of patients undergoing LTX after those undergoing transplantation due to chronic obstructive pulmonary disease (30\%). The ISHLT database shows the following percentages of cases undergoing LTx: chronic obstructive pulmonary disease, 36\%; IPF, 25\% [15]. From the United Network for Organ Sharing registry, which presents the comparison of pre-LAS and LAS lung transplant programmes, the conclusion can be drawn that LAS is a better system for patients with IPF. Before the use of LAS only $15 \%$ of patients with IPF in the USA underwent LTX, but after implementation of the LAS system, the percentage of lung recipients was $37 \%$ [10].

In conclusion, the 6-minute walk test distance is an independent predictor of mortality on the lung transplant waiting list. Patients should be referred to lung transplant centres for assessment when their 6-minute walk test distance is less than $350 \mathrm{~m}$, which may considerably increase their probability of receiving a lung graft. Blood type $A B$ is associated with a greater chance of receiving a graft, whereas blood type $O$ patients are more likely to die on the waiting list. Patients' height also plays a significant role in becoming a lung recipient. Due to the small size of the study group and its retrospective nature, the authors are aware of its limitations. 


\section{Conflict of interest}

The authors declare no conflict of interest.

\section{References}

1. Plantier L, Cazes A, Dinh-Xuan AT, et al. Physiology of the lung in idiopathic pulmonary fibrosis. Eur Respir Rev 2018; 27: pii:170062.

2. Sgalla G, lovene B, Calvello M, et al. Idiopathic pulmonary fibrosis: pathogenesis and management. Respir Res 2018; 19: 32.

3. Arundel C, Sheriff H, Bearden D, et al. Discharge home health services referral and 30-day all-cause readmission in older adults with heart failure. Arch Med Sci 2018; 14: 995-1002.

4. Scelfo C, Caminati A, Harari S. Recent advances in managing idiopathic pulmonary fibrosis. F1000Research 2017; 6: 2052

5. Grimm JC, Silhan LL, Shah AS. Lung transplantation for idiopathic pulmonary fibrosis. In: Lung Transplantation: Principles and Practice. Vigneswaran WT, Garrity ER, Odell JA (eds.). CRC Press, Boca Raton 2015; 183-9.

6. Zank DC, Bueno M, Mora AL, Rojas M. Idiopathic pulmonary fibrosis: aging, mitochondrial dysfunction, and cellular bioenergetic. Front Med 2018; 5: 10.

7. Baumgartner KB, Samet JM, Coultas DB, et al. Occupational and environmental risk factors for idiopathic pulmonary fibrosis: a multicenter case-control study. Collaborating Centers. Am J Epidemiol 2000; 152: 307.

8. Raghu G, Collard HR, Egan JJ, et al. An official ATS/ERS/ JRS/ALAT statement: idiopathic pulmonary fibrosis: evidence-based guidelines for diagnosis and management. Am J Respir Crit Care Med 2011; 183: 788-824.

9. Sgalla G, Biffi A, Richeldi L. Idiopathic pulmonary fibrosis: diagnosis, epidemiology and natural history. Respirology 2016; 21: 427-37.

10. George TJ, Arnaoutakis GJ, Shah AS. Lung transplant in idiopathic pulmonary fibrosis. Arch Surg 2011; 146 1204-9.

11. Weill D, Benden C, Corris PA, et al. A consensus document for the selection of lung transplant candidates: 2014 - An update from the Pulmonary Transplantation Council of the International Society for Heart and Lung Transplantation. J Heart Lung Transplant 2015; 34: 1-15.

12. Brown AW, Shlobin OA, Weir N, et al. Dynamic patient counseling: a novel concept in idiopathic pulmonary fibrosis. Chest 2012; 142: 1005-10.

13. Castria D, Refini RM, Bargagli E, et al. Pulmonary hypertension in idiopathic pulmonary fibrosis: prevalence and clinical progress. Int I Immunopathol Pharmacol 2012; 25: 681-9.

14. Mackay LS, Anderson RL, Parry G, et al. Pulmonary fibrosis: rate of disease progression as a trigger for referral for lung transplantation. Thorax 2007; 62: 1069-73.

15. Kułaga Z, Litwin M, Tkaczyk M, et al. Polish 2010 growth references for school-aged children and adolescents. Eur J Pediatr 2011; 170: 599-609.

16. Lederer DJ, Arcasoy SM, Wilt JS, et al. Six-minute-walk distance predicts waiting list survival in idiopathic pulmonary fibrosis. Am J Respir Crit Care Med 2006; 174: 659-64.

17. du Bois RM, Albera C, Bradford WZ, et al. 6-Minute walk distance is an independent predictor of mortality in patients with idiopathic pulmonary fibrosis. Eur Respir J 2014; 43: 1421-9.
18. Ikezoe K, Handa T, Tanizawa K, et al. Prognostic factors and outcomes in Japanese lung transplant candidates with interstitial lung disease. PLoS One 2017; 12: e0183171.

19. Bennett D, Fossi A, Bargagli E, et al. Mortality on the waiting list for lung transplantation in patients with idiopathic pulmonary fibrosis: a single-centre experience. Lung 2015; 193: 677-81.

20. Budzyński J, Tojek K, Wustrau B, et al. The "cholesterol paradox" among inpatients - retrospective analysis of medical documentation. Arch Med Sci Atheroscler Dis 2018; 3: 46-57. 